\title{
Effective Theories for Magnetic Systems
}

\author{
Uwe-Jens Wiese \\ Albert Einstein Center for Fundamental Physics \\ Institute for Theoretical Physics \\ Bern University, Sidlerstrasse 5, 3012 Bern, Switzerland \\ E-mail: wieseditp.unibe.ch
}

In complete analogy to chiral perturbation theory, systematic low-energy effective theories can be used to describe the lightly doped antiferromagnetic precursors of high-temperature superconductors. The spinwaves or magnons are the Goldstone bosons of the spontaneously broken $S U(2)_{s}$ symmetry. The comparison of analytic effective field theory results at the two-loop level and Monte Carlo data obtained with a very efficient cluster algorithm leads to a determination of the leading low-energy parameters with permille accuracy. While magnons are analogous to the pions in QCD, doped holes in an antiferromagnet are analogous to the nucleons. Flavor quantum numbers of doped holes emerge from non-trivial positions of the hole pockets in the Brillouin zone. Magnon-exchange leads to the formation of two-hole bound states analogous to the deuteron, and spiral phases of the staggered magnetization are a condensed matter analog of pion condensation in nuclear matter.

6th International Workshop on Chiral Dynamics, CD09

July 6-10, 2009

Bern, Switzerland 


\section{Introduction}

To a large extent, the success of theoretical physics is based on the concept of effective theories. In particular, there is no need to know the "theory of everything" before one can address the physics at a particular energy scale. Once the relevant degrees of freedom have been identified, and symmetry considerations are taken into account, the locality of space and time allows us to construct a systematic low-energy effective theory. This has been demonstrated in great detail in chiral perturbation theory - the systematic low-energy effective field theory description of the strong interaction. Chiral perturbation theory was originally developed for the lightest strongly interacting particles - the pseudo-Goldstone pions of the spontaneously broken $S U(2)_{L} \times S U(2)_{R}$ chiral symmetry of QCD [1, 2]. It was then extended to baryon chiral perturbation theory [3-6] which includes the nucleons as the lightest particles in the sector with baryon number one. Systematic effective theories have also been developed for few nucleon systems [7-10]. Thanks to asymptotic freedom, the fundamental QCD theory underlying all these effective theories is well-defined at arbitrarily high energy scales, and can hence be considered as the "theory of everything about the strong interaction". ${ }^{1}$ Given the value of the strong coupling constant as well as the quark masses (at some energy scale), QCD makes unique predictions for all processes of the strong interactions. The same physics is described by the corresponding effective theories, order by order in a systematic low-energy expansion. The effective theories contain a large number of a priori undetermined low-energy parameters. Matching these parameters to the underlying QCD theory is an important incentive of lattice QCD, the nonperturbative first principles approach to the underlying fundamental theory itself. Although lattice QCD will eventually undoubtedly solve QCD with high precision, the corresponding systematic low-energy effective theories will always remain extremely valuable because they add tremendous analytic understanding to the numbers produced by lattice QCD. The strong interactions provide a perfect example for how the interplay between the underlying fundamental theory and the corresponding low-energy effective theories can advance our understanding of non-trivial dynamical phenomena.

Highly non-trivial dynamics is at work also in condensed matter physics. In particular, understanding high-temperature superconductivity [11] remains one of the greatest challenges in condensed matter physics. Unlike in particle physics, there is no general agreement about what the underlying "theory of everything about high-temperature superconductivity" should be. Still, most experts agree that some variant of the Hubbard or $t-J$ model should capture the relevant microscopic physics. Just like solving lattice QCD, it is a tremendous numerical and algorithmic challenge to solve these microscopic models addressing high-temperature superconductivity. In particular, at non-zero doping (which is analogous to non-zero baryon density in QCD) numerical simulations suffer from very severe sign problems. Even the lightly doped antiferromagnetic precursors of high-temperature superconductors possess a highly non-trivial dynamics and pose great theoretical challenges. Using a variety of experimental and theoretical methods, a lot has been learned about these systems. In particular, at zero doping the relevant degrees of freedom are the magnon Goldstone bosons of the $S U(2)_{s}$ symmetry, which is spontaneously broken to a $U(1)_{s}$ subgroup by the formation of the staggered magnetization order parameter characteristic for antiferromagnetism. In complete analogy to pion chiral perturbation theory, systematic low-energy effective field theories

\footnotetext{
${ }^{1}$ Obviously, QCD is embedded in the Standard model, which — due to its triviality — is only an effective theory.
} 


\begin{tabular}{|c|c|c|}
\hline & QCD & Antiferromagnetism \\
\hline \hline broken phase & hadronic vacuum & antiferromagnetic phase \\
\hline global symmetry & chiral symmetry & spin rotations \\
\hline symmetry group $G$ & $S U(2)_{L} \times S U(2)_{R}$ & $S U(2)_{s}$ \\
\hline unbroken subgroup $H$ & $S U(2)_{L=R}$ & $U(1)_{s}$ \\
\hline Goldstone boson & pion & magnon \\
\hline Goldstone field in $G / H$ & $U(x) \in S U(2)$ & $\vec{e}(x) \in S^{2}$ \\
\hline order parameter & chiral condensate & staggered magnetization $\mathscr{M}_{s}$ \\
\hline coupling strength & pion decay constant & spin stiffness $\rho_{s}$ \\
\hline propagation speed & velocity of light & spinwave velocity $c$ \\
\hline conserved charge & baryon number $U(1)_{B}$ & electric charge $U(1)_{Q}$ \\
\hline charged particle & nucleon or antinucleon & electron or hole \\
\hline long-range force & pion exchange & magnon exchange \\
\hline inhomogeneous phase & spiral phase & pion condensate \\
\hline dense phase & nuclear or quark matter & high- $T_{c}$ superconductor \\
\hline microscopic description & lattice QCD & Hubbard or $t$ - $J$ model \\
\hline $\begin{array}{c}\text { effective description } \\
\text { of Goldstone bosons }\end{array}$ & chiral perturbation & magnon effective \\
\hline $\begin{array}{c}\text { effective description } \\
\text { of charged fields }\end{array}$ & $\begin{array}{c}\text { baryon chiral } \\
\text { perturbation theory }\end{array}$ & $\begin{array}{c}\text { magnon-hole } \\
\text { effective theory }\end{array}$ \\
\hline
\end{tabular}

Table 1: Some analogies between QCD and antiferromagnetism.

have been developed for magnons both in ferro- and in antiferromagnets [12-19]. In fact, thanks to the interplay between analytic calculations in effective field theory and accurate numerical simulations [20,21], the undoped antiferromagnetic precursors of high-temperature superconductors like $\mathrm{La}_{2} \mathrm{CuO}_{4}$ and $\mathrm{Sr}_{2} \mathrm{CuO}_{2} \mathrm{Cl}_{2}$ are among the quantitatively best understood condensed matter systems.

At low doping, in addition to the magnons, doped holes enter as relevant low-energy degrees of freedom. The dynamics of doped holes has also been addressed with effective theories [22-25]. However, there has been no agreement on important issues including the transformation rules of the hole fields under the various symmetries. In complete analogy to baryon chiral perturbation theory, and based on experimental and numerical results [26, 27] for the underlying microscopic systems, fully systematic low-energy effective field theories for lightly doped antiferromagnets have been constructed in [28-33]. Several of these theories will be discussed below. Some analogies between QCD and antiferromagnetism are listed in table 1.

\section{Undoped Antiferromagnets}

In this section we discuss effective theories for undoped antiferromagnets. A concrete underlying microscopic system is the quantum Heisenberg model with the Hamiltonian

$$
H=J \sum_{x, i} \vec{S}_{x} \cdot \vec{S}_{x+\hat{i}}, \quad\left[S_{x}^{a}, S_{y}^{b}\right]=i \delta_{x y} \varepsilon_{a b c} S_{x}^{c} .
$$


Here $x$ denotes sites on a 2-dimensional bipartite (e.g. square or honeycomb) lattice with spacing $a$, and $\hat{i}$ is a vector of length $a$ in a lattice direction. The spin $\frac{1}{2}$ operators $\vec{S}_{x}$ obey the standard $S U(2)_{s}$ commutation relations. Note that we work in natural units in which $\hbar=1$. The Hamiltonian commutes with the total spin $\vec{S}=\sum_{x} \vec{S}_{x}$ and is thus invariant under $S U(2)_{s}$ spin rotations. As one has learned from detailed numerical simulations, at zero temperature the $S U(2)_{s}$ symmetry is spontaneously broken down to a $U(1)_{s}$ subgroup, both on a square and on a honeycomb lattice.

The corresponding low-energy effective field theory is formulated in terms of the staggered magnetization order parameter field

$$
\vec{e}(x)=\left(e_{1}(x), e_{2}(x), e_{3}(x)\right) \in S^{2}, \quad \vec{e}(x)^{2}=1,
$$

which takes values in the coset space $S U(2)_{s} / U(1)_{s}=S^{2}$. Here $x=\left(x_{1}, x_{2}, t\right)$ is a point in Euclidean space-time. The leading terms in the effective action for the staggered magnetization field take the form

$$
S[\vec{e}]=\int d^{2} x d t \frac{\rho_{s}}{2}\left(\partial_{i} \vec{e} \cdot \partial_{i} \vec{e}+\frac{1}{c^{2}} \partial_{t} \vec{e} \cdot \partial_{t} \vec{e}\right)
$$

where $\rho_{s}$ is the spin stiffness. Antiferromagnetic magnons have a "relativistic" dispersion relation with the spinwave velocity $c$ playing the role of the velocity of light. It should be pointed out that Euclidean rotation invariance is just an accidental symmetry of the leading terms of the effective action.

It is interesting to note that the ferromagnetic quantum Heisenberg model (which differs from the antiferromagnet only by the sign of the Hamiltonian) has rather different symmetry properties at low energies. Unlike quantum antiferromagnets, quantum ferromagnets have a conserved order parameter - the uniform magnetization. Consequently, ferromagnetic magnons have a nonrelativistic dispersion relation and the corresponding effective action contains an additional Wess-Zumino term [18], which breaks rotation invariance between space and Euclidean time already at leading order. The resulting effective field theory for ferromagnetic magnons has been studied in $[18,19]$.

\subsection{Determination of the Low-Energy Parameters in the Cubic $\varepsilon$-Regime}

Hasenfratz and Niedermayer have used the effective theory to derive the finite-size and finitetemperature effects of the staggered susceptibility

$$
\chi_{s}=\frac{\mathscr{M}_{s}^{2} L^{2} \beta}{3}\left\{1+2 \frac{c}{\rho_{s} L l} \beta_{1}(l)+\left(\frac{c}{\rho_{s} L l}\right)^{2}\left[\beta_{1}(l)^{2}+3 \beta_{2}(l)\right]+\mathscr{O}\left(\frac{1}{L^{3}}\right)\right\}
$$

from a 2-loop calculation in the $\varepsilon$-regime of magnon chiral perturbation theory [17]. Here $\mathscr{M}_{s}$ is the staggered magnetization density. Similarly, the uniform susceptibility takes the form

$$
\chi_{u}=\frac{2 \rho_{s}}{3 c^{2}}\left\{1+\frac{1}{3} \frac{c}{\rho_{s} L l} \widetilde{\beta}_{1}(l)+\frac{1}{3}\left(\frac{c}{\rho_{s} L l}\right)^{2}\left[\widetilde{\beta}_{2}(l)-\frac{1}{3} \widetilde{\beta}_{1}(l)^{2}-6 \psi(l)\right]+\mathscr{O}\left(\frac{1}{L^{3}}\right)\right\} .
$$

Here $l=(\beta c / L)^{1 / 3}$ determines the shape of an approximately cubic space-time box of size $L \times L \times$ $\beta$, with $\beta c \approx L$. The functions $\beta_{i}(l), \widetilde{\beta}_{i}(l)$, and $\psi(l)$ are known shape-coefficients $[15,17]$.

The susceptibilities $\chi_{s}$ and $\chi_{u}$ have been calculated numerically for the antiferromagnetic spin $\frac{1}{2}$ quantum Heisenberg model on the square lattice using a very efficient loop-cluster algorithm 
[20,34]. In a recent study using a zero-temperature valence-bond projector method, a very accurate result was obtained for the staggered magnetization per spin $\widetilde{\mathscr{M}}_{s}=\mathscr{M}_{s} a^{2}$ [35]. The best estimate of the low-energy parameters is given by [36]

$$
\widetilde{\mathscr{M}_{s}}=0.30743(1), \quad \rho_{s}=0.1808(4) J, \quad c=1.6585(10) J a \quad \text { (square lattice) } .
$$

In addition to the cuprates, another superconducting material, $\mathrm{Na}_{x} \mathrm{CoO}_{2} \cdot \mathrm{yH}_{2} \mathrm{O}$, has drawn a lot of attention both theoretically and experimentally. Unfortunately, due to the fact that the underlying lattice geometry of the spin $\frac{1}{2}$ cobalt sites in these materials is triangular - which leads to strong geometric frustration - a first principles Monte Carlo study is impossible in practice. Nevertheless, at filling $x=\frac{1}{3}$ the unhydrated parent compound $\mathrm{Na}_{x} \mathrm{CoO}_{2}$ may be described by the Heisenberg model on a honeycomb lattice which allows one to simulate the system efficiently with the loop-cluster algorithm. In this case one obtains [37]

$$
\widetilde{\mathscr{M}_{s}}=0.2688(3), \quad \rho_{s}=0.102(2) J, \quad c=1.297(16) J a \quad \text { (honeycomb lattice) }
$$

The reduction of the staggered magnetization per spin $\widetilde{\mathscr{M}}_{s}$ and the spin stiffness $\rho_{s}$ compared to the square lattice case indicates larger quantum fluctuations on the honeycomb lattice. This is expected since the coordination number of the honeycomb lattice is smaller than the one of the square lattice. Once the low-energy parameters are determined with high precision, the effective theory makes unambiguous predictions, which in turn are testable in numerical simulations.

\subsection{Rotor Spectrum in the Cylindrical $\delta$-Regime}

In the very low temperature limit, one enters the cylindrical $\delta$-regime of space-time volumes with $\beta c \gg L$. In this case, the staggered magnetization vector acts as a quantum rotor and, correspondingly, the low-energy end of the spectrum takes the form $E_{S}=S(S+1) / 2 \Theta$. Here $S \in\{0,1,2, \ldots\}$ is the total spin and $\Theta$ is the moment of inertia of the quantum rotor which is given by [17]

$$
\Theta=\frac{\rho_{s} L^{2}}{c^{2}}\left[1+\frac{3.900265 c}{4 \pi \rho_{s} L}+\mathscr{O}\left(\frac{1}{L^{2}}\right)\right] .
$$

The probability distribution of the uniform magnetization $M^{3}=S^{3}$ takes the form

$$
p\left(M^{3}\right)=\frac{1}{Z} \sum_{S \geq\left|M^{3}\right|} \exp \left(-\beta E_{S}\right), \quad Z=\sum_{S=0}^{\infty}(2 S+1) \exp \left(-\beta E_{S}\right) .
$$

Having determined the values of the low-energy parameters $\widetilde{\mathscr{M}_{s}}, \rho_{s}$, and $c$ from the cubic space-time regime, one can now test the effective theory in the cylindrical regime. Figure 1 shows a comparison of the effective theory prediction for the probability distribution $p\left(M^{3}\right)$ of eq.(2.9) with Monte Carlo data for the system on the honeycomb lattice. The observed excellent agreement - which does not involve any adjustable parameters - confirms the quantitative correctness of the effective theory. 

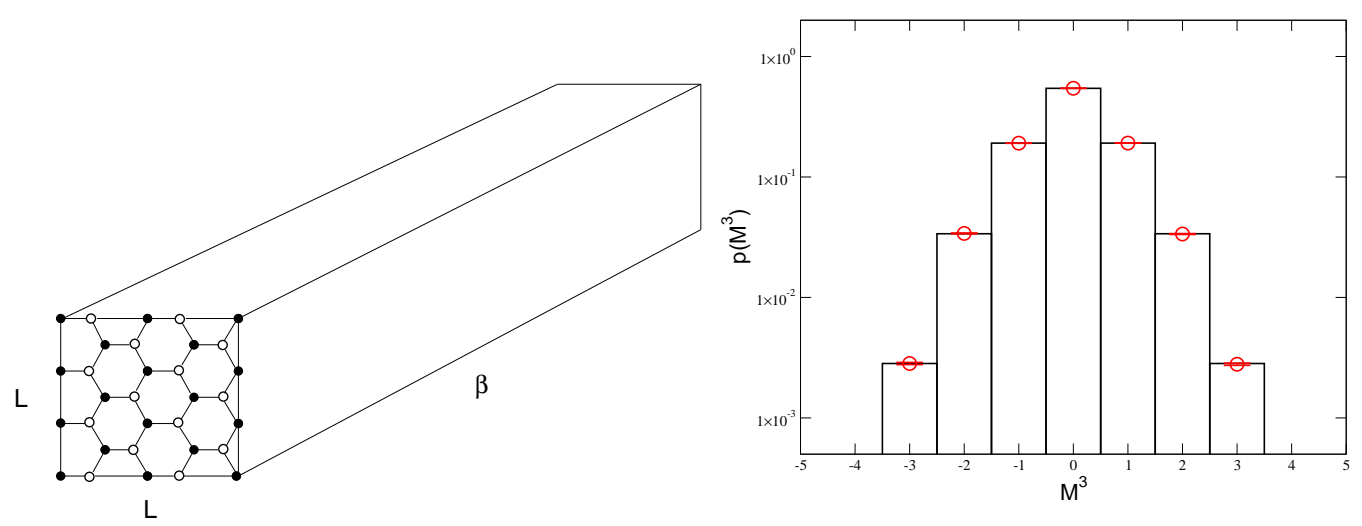

Figure 1: Cylindrical space-time volume for a honeycomb lattice (left). Comparison of the effective theory prediction for the probability distribution $p\left(M^{3}\right)$ with Monte Carlo data in the cylindrical $\delta$-regime on a honeycomb lattice (right).

\subsection{Constraint Effective Potential for the Square Lattice Antiferromagnet}

Let us again consider the system in a periodic cubic space-time volume $L \times L \times \beta$ with the inverse temperature fixed at $\beta=L / c$. The space-time average of the staggered magnetization is given by

$$
\vec{\Phi}=\frac{1}{2} \frac{1}{L^{2} \beta} \int d^{2} x d t \vec{e}(x)=\frac{1}{2} \frac{1}{L^{3}} \int d^{3} x \vec{e}(x) .
$$

Due to the $S U(2)_{s}$ symmetry, the probability distribution

$$
p(\Phi)=\frac{1}{Z} \int D \vec{e} \exp (-S[\vec{e}]) \delta\left(\vec{\Phi}-\frac{1}{2} \frac{1}{L^{3}} \int d^{3} x \vec{e}(x)\right)=\mathscr{N} \exp (-U(\Phi)) .
$$

of the mean staggered magnetization vector $\vec{\Phi}$ only depends on the magnitude $\Phi=|\vec{\Phi}|$. The constraint effective potential $U(\Phi)$ represents the free energy density of configurations constrained to a fixed mean staggered magnetization $\Phi$. Göckeler and Leutwyler have used chiral perturbation theory to systematically work out the finite-size effects of the constraint effective potential near its minimum [38, 39]. At leading order, $U(\Phi)=U_{0}(\psi)$ is a known universal function of the rescaled variable $\psi=\rho_{s} L\left(\Phi-\widetilde{\mathscr{M}_{s}}\right) / \widetilde{\mathscr{M}}_{s} c$. Some values of the function $U_{0}(\psi)$ extracted from the numerical data of [36] are compared with the analytic result of [38, 39] in figure 2. It should be pointed out that the observed perfect agreement does not depend on any adjustable parameters, and thus again confirms the correctness of the effective theory in great detail.

\section{Lightly Doped Antiferromagnets}

The standard microscopic models for antiferromagnetism and high-temperature superconductivity are Hubbard and $t-J$-type models. The symmetries of these models are of central importance for the construction of the low-energy effective theories for magnons and holes. The $t-J$ model is defined by the nearest-neighbor hopping Hamilton operator

$$
H=P\left\{-t \sum_{x, i}\left(c_{x}^{\dagger} c_{x+\hat{i}}+c_{x+\hat{i}}^{\dagger} c_{x}\right)+J \sum_{x, i} \vec{S}_{x} \cdot \vec{S}_{x+\hat{i}}\right\} P, \quad c_{x}=\left(\begin{array}{c}
c_{x \uparrow} \\
c_{x \downarrow}
\end{array}\right), \quad \vec{S}_{x}=c_{x}^{\dagger} \frac{\vec{\sigma}}{2} c_{x} .
$$



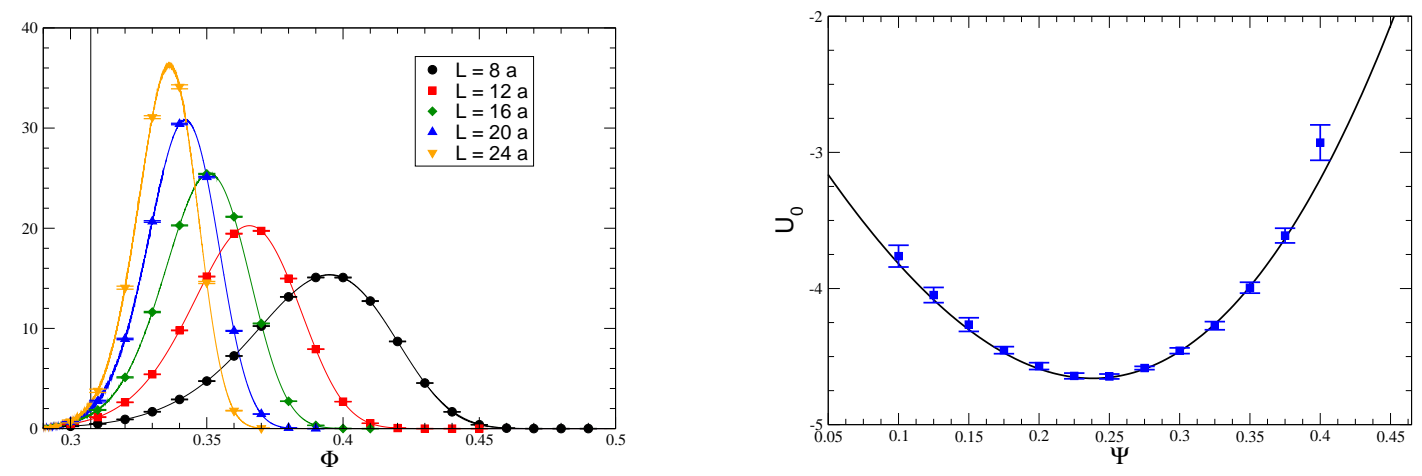

Figure 2: Probability distributions $p(\Phi)$ of the mean staggered magnetization on the square lattice for different volumes (left). In the infinite-volume limit the finite-volume curves approach the vertical line which marks the order parameter $\widetilde{\mathscr{M}}_{s}=0.30743(1)$. The analytic result for the universal function $U_{0}(\psi)$ is compared to Monte Carlo data without any adjustable parameters (right).
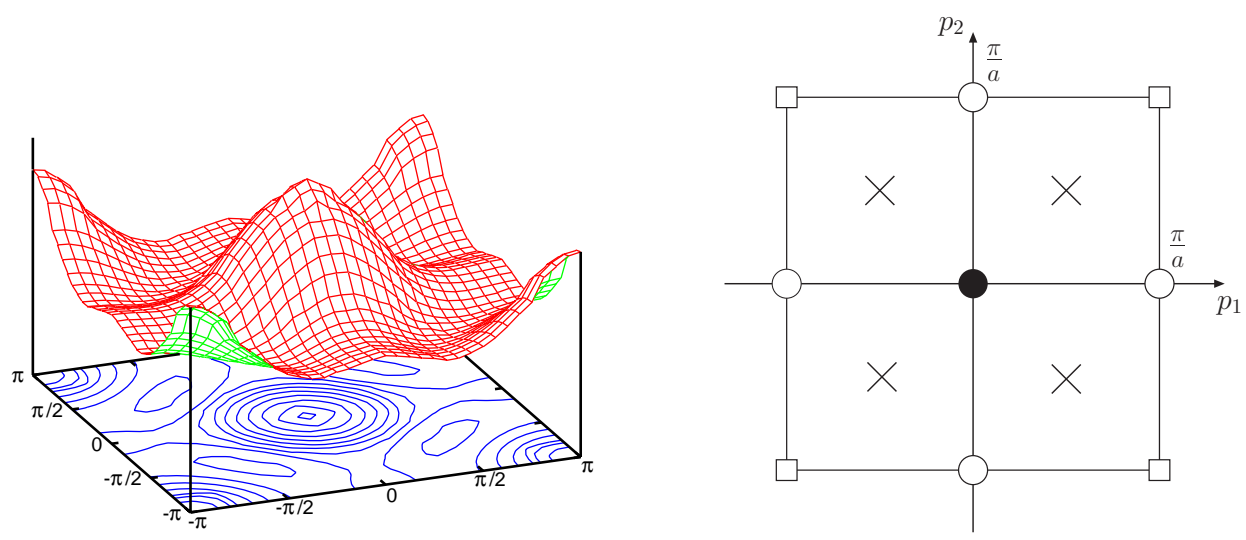

Figure 3: The energy-momentum dispersion relation for a single hole in the $t-J$ model on a square lattice over the corresponding Brillouin zone (left). The holes reside in momentum space pockets centered at lattice momenta $\left( \pm \frac{\pi}{2 a}, \pm \frac{\pi}{2 a}\right)$ which are represented by the four crosses (right).

The fermion creation and annihilation operators obey standard anticommutation relations and act in a restricted Hilbert space of empty or at most singly occupied sites, while states with doubly occupied sites are eliminated from the Hilbert space by the projection operator $P$. The $t-J$ model is invariant against $S U(2)_{s}$ spin rotations, $U(1)_{Q}$ fermion number transformations, the discrete rotations, reflections, and shift symmetries of the spatial lattice, as well as against time-reversal. At zero doping, i.e. at half-filling, the $t-J$ model reduces to the Heisenberg model.

As illustrated in figure 3, numerical simulations of the single-hole sector of the $t-J$ model show that the holes reside in momentum-space pockets centered at $\left( \pm \frac{\pi}{2 a}, \pm \frac{\pi}{2 a}\right)$ in the Brillouin zone $[26,27,30]$. There are four half-pockets which give rise to two different species of holes. In the effective theory the location of the two species in different regions of the Brillouin zone manifests itself as a flavor index that responds to discrete rotations, reflections, and shift symmetries. 


\subsection{Nonlinear Realization of the $S U(2)_{s}$ Symmetry}

In order to couple the holes to the magnons, a nonlinear realization of the $S U(2)_{s}$ symmetry has been constructed in [28]. The global $S U(2)_{s}$ symmetry then manifests itself as a local $U(1)_{s}$ symmetry in the unbroken subgroup. This is analogous to baryon chiral perturbation theory in which the spontaneously broken $S U(2)_{L} \times S U(2)_{R}$ chiral symmetry of QCD is implemented on the nucleon fields as a local $S U(2)_{L=R}$ transformation in the unbroken isospin subgroup. The definition of the nonlinear realization of the $S U(2)_{s}$ symmetry proceeds as follows. First, one diagonalizes the magnon field by a unitary transformation $u(x) \in S U(2)_{s}$, i.e.

$$
u(x) \frac{1}{2}(\mathbb{1}+\vec{e}(x) \cdot \vec{\sigma}) u(x)^{\dagger}=\frac{1}{2}\left(\mathbb{1}+\sigma_{3}\right)=\left(\begin{array}{ll}
1 & 0 \\
0 & 0
\end{array}\right), \quad u_{11}(x) \geq 0 .
$$

Under an $S U(2)_{s}$ transformation $g$, the diagonalizing field $u(x)$ transforms as $u(x)^{\prime}=h(x) u(x) g^{\dagger}$, which implicitly defines the nonlinear symmetry transformation $h(x) \in U(1)_{s}$. The traceless antiHermitean field

$$
v_{\mu}(x)=u(x) \partial_{\mu} u(x)^{\dagger}, \quad v_{\mu}(x)=i v_{\mu}^{a}(x) \sigma_{a}, \quad v_{\mu}^{ \pm}(x)=v_{\mu}^{1}(x) \mp i v_{\mu}^{2}(x),
$$

decomposes into an Abelian "gauge" field $v_{\mu}^{3}(x)$ and two "charged" vector fields $v_{\mu}^{ \pm}(x)$ to which the doped holes can couple.

\subsection{Effective Lagrangian for Magnons and Holes on the Square Lattice}

The effective field theory is defined in the space-time continuum and the holes are described by two independent Grassmann-valued fields $\psi_{s}^{f}(x)$ and $\psi_{s}^{f \dagger}(x)$ carrying a "flavor" index $f=\alpha, \beta$ that characterizes the corresponding hole pocket. The index $s= \pm$ denotes spin parallel ( + ) or antiparallel (-) to the local staggered magnetization. A detailed symmetry analysis was carried out in [30]. The resulting leading terms in the effective Lagrangian with two fermion fields (containing at most one temporal or two spatial derivatives) describe the propagation of holes as well as their couplings to magnons and are given by

$$
\begin{aligned}
& \mathscr{L}_{2}=\sum_{\substack{f=\alpha, \beta \\
s=+,-}}\left[M \psi_{s}^{f \dagger} \psi_{s}^{f}+\psi_{s}^{f \dagger} D_{t} \psi_{s}^{f}+\frac{1}{2 M^{\prime}} D_{i} \psi_{s}^{f \dagger} D_{i} \psi_{s}^{f}+\sigma_{f} \frac{1}{2 M^{\prime \prime}}\left(D_{1} \psi_{s}^{f \dagger} D_{2} \psi_{s}^{f}+D_{2} \psi_{s}^{f \dagger} D_{1} \psi_{s}^{f}\right)\right. \\
& \left.+\Lambda\left(\psi_{s}^{f \dagger} v_{1}^{s} \psi_{-s}^{f}+\sigma_{f} \psi_{s}^{f \dagger} v_{2}^{s} \psi_{-s}^{f}\right)+N_{1} \psi_{s}^{f \dagger} v_{i}^{s} v_{i}^{-s} \psi_{s}^{f}+\sigma_{f} N_{2}\left(\psi_{s}^{f \dagger} v_{1}^{s} v_{2}^{-s} \psi_{s}^{f}+\psi_{s}^{f \dagger} v_{2}^{s} v_{1}^{-s} \psi_{s}^{f}\right)\right] .
\end{aligned}
$$

Here $M$ is the rest mass and $M^{\prime}$ and $M^{\prime \prime}$ are the kinetic masses of a hole, $\Lambda$ is a hole-one-magnon, and $N_{1}$ and $N_{2}$ are hole-two-magnon couplings, which all take real values. The sign $\sigma_{f}$ is + for $f=\alpha$ and - for $f=\beta$. The covariant derivatives are given by $D_{\mu} \psi_{ \pm}^{f}(x)=\left[\partial_{\mu} \pm i v_{\mu}^{3}(x)\right] \psi_{ \pm}^{f}(x)$. Remarkably, the term in the Lagrangian proportional to $\Lambda$ contains just a single (uncontracted) spatial derivative (which is contained in $v_{i}^{S}$ ). Due to the nontrivial rotation properties of flavor, this term is still 90 degrees rotation invariant. Due to the small number of derivatives it contains, this term dominates the low-energy dynamics. In particular, it alone is responsible for one-magnon exchange and for the existence of spiral phases. The QCD analog of $\Lambda$ is the coupling $g_{A}$. 


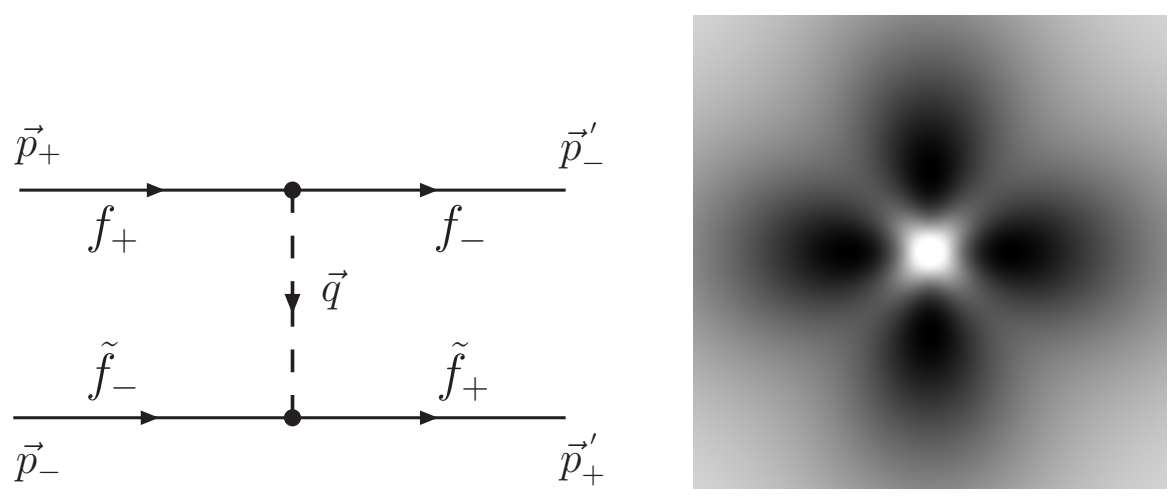

Figure 4: One-magnon exchange between two holes (left), and probability distribution for the bound state of two holes with flavors $\alpha$ and $\beta$ (right).

\subsection{Magnon-mediated Two-Hole Bound States}

Figure 4 shows the Feynman diagram for one-magnon exchange between two holes. Depending on the flavor-combination, the resulting potentials are given by

$$
V^{\alpha \alpha}(\vec{r})=\gamma \frac{\sin (2 \varphi)}{r^{2}}, \quad V^{\beta \beta}(\vec{r})=-\gamma \frac{\sin (2 \varphi)}{r^{2}}, \quad V^{\alpha \beta}(\vec{r})=V^{\beta \alpha}(\vec{r})=\gamma \frac{\cos (2 \varphi)}{r^{2}} .
$$

where $\gamma=\Lambda^{2} / 2 \pi \rho_{s}$. Here $\vec{r}$ is the distance vector between the two holes and $\varphi$ is the angle between $\vec{r}$ and a lattice axis. The corresponding two-hole Schrödinger equation has been solved analytically in [30]. Figure 4 also illustrates the resulting probability distribution for the bound state of two holes with flavors $\alpha$ and $\beta$. Although it seems to resemble $d_{x^{2}-y^{2}}$ symmetry, it actually has p-wave symmetry. Since the Cooper pairs of high-temperature superconductors have d-wave symmetry, one may conclude that magnon-mediated two-hole bound states in a lightly doped antiferromagnet do not resemble Cooper pairs. They are, however, a condensed matter analog of the deuteron.

\subsection{Spiral Phases of the Staggered Magnetization}

The systematic effective field theory for antiferromagnetic magnons and holes has also been used to investigate the propagation of holes in the background of a spatially varying staggered magnetization field [31]. For large values of $\rho_{s}$, distortions in the staggered magnetization cost a large amount of energy and a homogeneous phase is energetically favored. In that case, all four hole pockets are equally populated with doped holes. For smaller values of $\rho_{s}$, on the other hand, the doped holes can gain energy from a spiral in the staggered magnetization. For intermediate values of $\rho_{s}$ a zero degree spiral is realized, in which only two hole pockets are populated. The homogeneous as well as the spiral phase are illustrated in figure 5. It should be noted that spiral phases arise due to the leading magnon-hole coupling $\Lambda$. In electron-doped antiferromagnets the charge carriers reside in other places in the Brillouin zone. As a consequence, an analog of the $\Lambda$-term is absent in the electron-doped case, and spiral phases do not arise [32]. Spiral phases have indeed been observed in certain hole- but not in electron-doped antiferromagnets. They are a condensed matter analog of pion condensates in nuclear matter. 

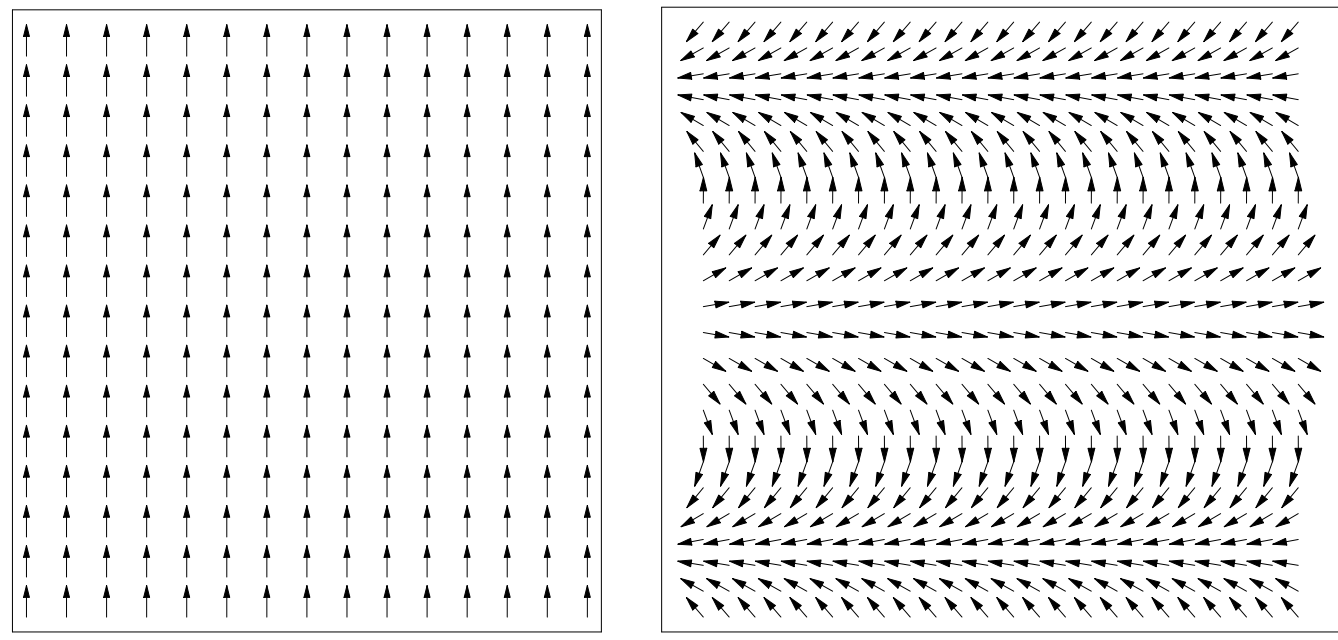

Figure 5: The homogeneous phase with constant staggered magnetization (left) as well as a zero degree spiral oriented along a lattice axis (right).
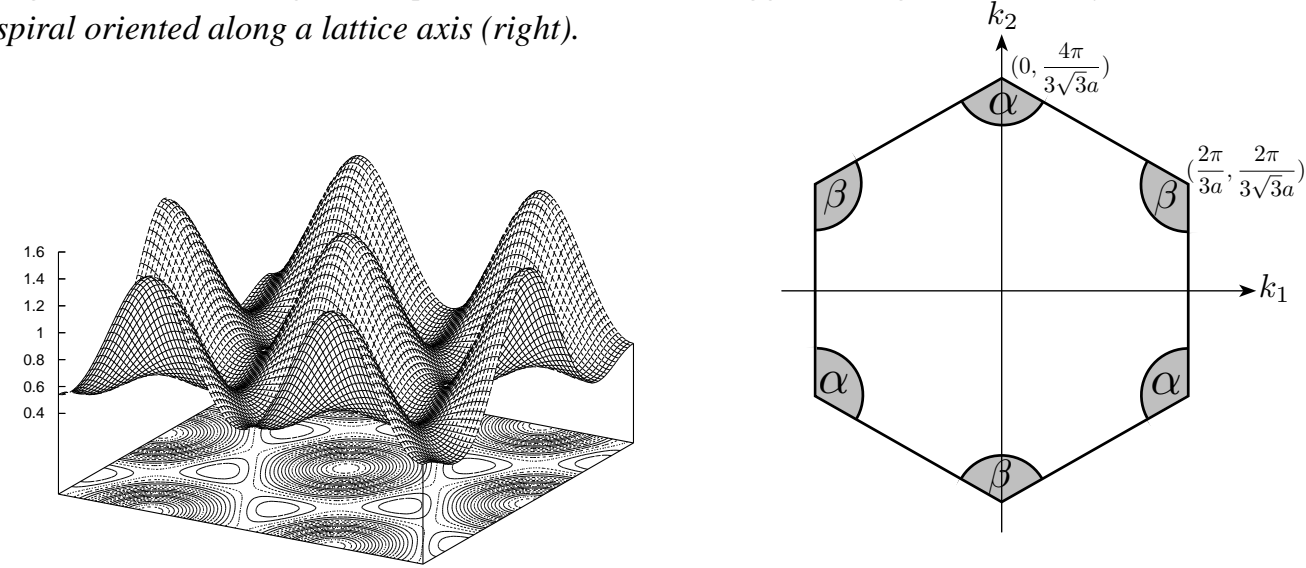

Figure 6: The energy-momentum dispersion relation for a single hole in the $t$-J model on a honeycomb lattice over the corresponding Brillouin zone (left). The holes reside in momentum space pockets centered at lattice momenta at the zone corners (right).

\subsection{Effective Lagrangian for Holes on the Honeycomb Lattice}

The single hole sector of the $t-J$ model on the honeycomb lattice has been investigated in [37]. The resulting dispersion relation is illustrated in figure 6 . Again, in the effective continuum theory the location of holes in lattice momentum space manifests itself as a flavor index. The leading terms of the effective Lagrangian with two fermion fields (containing at most one temporal or two spatial derivatives) are given by

$$
\begin{aligned}
\mathscr{L}_{2} & =\sum_{\substack{f=\alpha, \beta \\
s=+,-}}\left[M \psi_{s}^{f \dagger} \psi_{s}^{f}+\psi_{s}^{f \dagger} D_{t} \psi_{s}^{f}+\frac{1}{2 M^{\prime}} D_{i} \psi_{s}^{f \dagger} D_{i} \psi_{s}^{f}+\Lambda \psi_{s}^{f \dagger}\left(i s v_{1}^{s}+\sigma_{f} v_{2}^{s}\right) \psi_{-s}^{f}\right. \\
& +i K\left[\left(D_{1}+i s \sigma_{f} D_{2}\right) \psi_{s}^{f \dagger}\left(v_{1}^{s}+i s \sigma_{f} v_{2}^{s}\right) \psi_{-s}^{f}-\left(v_{1}^{s}+i s \sigma_{f} v_{2}^{s}\right) \psi_{s}^{f \dagger}\left(D_{1}+i s \sigma_{f} D_{2}\right) \psi_{-s}^{f}\right] \\
& \left.+\sigma_{f} L \psi_{s}^{f \dagger} \varepsilon_{i j} f_{i j}^{3} \psi_{s}^{f}+N_{1} \psi_{s}^{f \dagger} v_{i}^{s} v_{i}^{-s} \psi_{s}^{f}+i s \sigma_{f} N_{2}\left(\psi_{s}^{f \dagger} v_{1}^{s} v_{2}^{-s} \psi_{s}^{f}-\psi_{s}^{f \dagger} v_{2}^{s} v_{1}^{-s} \psi_{s}^{f}\right)\right] .
\end{aligned}
$$


Here $M$ is the rest mass and $M^{\prime}$ is the kinetic mass of a hole, $\Lambda$ is the leading and $K$ is a sub-leading hole-one-magnon coupling, while $L, N_{1}$, and $N_{2}$ are hole-two-magnon couplings, and $f_{i j}^{3}(x)=$ $\partial_{i} v_{j}^{3}(x)-\partial_{j} v_{i}^{3}(x)$ is the field strength of the composite Abelian "gauge" field. Due to the singlederivative coupling $\Lambda$, spiral phases arise in this case as well [33].

\section{Conclusions}

Both for the strong interactions and for lightly doped antiferromagnets systematic low-energy effective field theories provide valuable analytic insight into the highly non-trivial dynamics, as well as accurate predictions depending on a number of a priori undetermined low-energy parameters. Using very efficient cluster algorithms, for quantum antiferromagnets some of these parameters have been determined with permille accuracy. The results presented here should also be encouraging for lattice QCD simulations, were the numerical problem is much harder. Eventually, one may expect agreement between lattice QCD and chiral perturbation theory at the same level of accuracy as achieved in the condensed matter problems discussed here.

\section{Acknowledgements}

The results presented here have been obtained in collaboration with C. Brügger, U. Gerber, C. P. Hofmann, F.-J. Jiang, F. Kämpfer, M. Nyfeler, M. Moser, and M. Pepe, whom I thank for a very pleasant collaboration. This work is supported in part by funds provided by the Schweizerischer Nationalfonds (SNF). The "Albert Einstein Center for Fundamental Physics" at Bern University is supported by the "Innovations- und Kooperationsprojekt C-13" of the Schweizerische Universitätskonferenz (SUK/CRUS).

\section{References}

[1] S. Weinberg, Physica 96 A (1979) 327.

[2] J. Gasser and H. Leutwyler, Annals Phys. 158 (1984) 142.

[3] J. Gasser, M.-E. Sainio, and A. Svarc, Nucl. Phys. B307 (1988) 779.

[4] E. Jenkins and A. Manohar, Phys. Lett. B255 (1991) 558.

[5] V. Bernard, N. Kaiser, J. Kambor, and U.-G. Meissner, Nucl. Phys. B388 (1992) 315.

[6] T. Becher and H. Leutwyler, Eur. Phys. J. C9 (1999) 643.

[7] S. Weinberg, Phys. Lett. B251 (1990) 288; Nucl. Phys. B363 (1991) 3; Phys. Lett. B295 (1992) 114.

[8] D. B. Kaplan, M. J. Savage, and M. B. Wise, Phys. Lett. B424 (1998) 390; Nucl. Phys. B534 (1998) 329.

[9] E. Epelbaum, W. Glöckle, and U.-G. Meissner, Nucl. Phys. A637 (1998) 107; Nucl. Phys. A684 (2001) 371; Nucl. Phys. A714 (2003) 535. 
[10] P. F. Bedaque, H.-W. Hammer, and U. van Kolck, Phys. Rev. C58 (1998) 641; Phys. Rev. Lett. 82 (1999) 463; Nucl. Phys. A676 (2000) 357.

[11] J. C. Bednorz and K. A. Müller, Z. Phys. B64 (1986) 189.

[12] S. Chakravarty, B. I. Halperin, and D. R. Nelson, Phys. Rev. B39 (1989) 2344.

[13] H. Neuberger and T. Ziman, Phys. Rev. B39 (1989) 2608.

[14] D. S. Fisher, Phys. Rev. B39 (1989) 11783.

[15] P. Hasenfratz and H. Leutwyler, Nucl. Phys. B343 (1990) 241.

[16] P. Hasenfratz and F. Niedermayer, Phys. Lett. B268 (1991) 231.

[17] P. Hasenfratz and F. Niedermayer, Z. Phys. B92 (1993) 91.

[18] H. Leutwyler, Phys. Rev. D49 (1994) 3033.

[19] C. P. Hofmann, Phys. Rev. B60 (1999) 388; Phys. Rev. B60 (1999) 406; cond-mat/0106492; cond-mat/0202153.

[20] U.-J. Wiese and H.-P. Ying, Z. Phys. B93 (1994) 147.

[21] B. B. Beard and U.-J. Wiese, Phys. Rev. Lett. 77 (1996) 5130.

[22] B. I. Shraiman and E. D. Siggia, Phys. Rev. Lett. 60 (1988) 740; Phys. Rev. Lett. 61 (1988) 467; Phys. Rev. Lett. 62 (1989) 1564; Phys. Rev. B46 (1992) 8305.

[23] X. G. Wen, Phys. Rev. B39 (1989) 7223.

[24] R. Shankar, Phys. Rev. Lett. 63 (1989) 203; Nucl. Phys. B330 (1990) 433.

[25] M. Y. Kuchiev and O. P. Sushkov, Physica C218 (1993) 197.

[26] M. Brunner, F. F. Assaad, and A. Muramatsu, Phys. Rev. B62 (2000) 15480.

[27] A. S. Mishchenko, N. V. Prokof'ev, and B. V. Svistunov, Phys. Rev. B64 (2001) 033101.

[28] F. Kämpfer, M. Moser, and U.-J. Wiese, Nucl. Phys. B729 (2005) 317.

[29] C. Brügger, F. Kämpfer, M. Pepe, and U.-J. Wiese, Eur. Phys. J. B53 (2006) 433.

[30] C. Brügger, F. Kämpfer, M. Moser, M. Pepe, and U.-J. Wiese, Phys. Rev. B74 (2006) 224432.

[31] C. Brügger, C. P. Hofmann, F. Kämpfer, M. Pepe, and U.-J. Wiese, Phys. Rev. B75 (2007) 014421.

[32] C. Brügger, C. P. Hofmann, F. Kämpfer, M. Moser, M. Pepe, and U.-J. Wiese, Phys. Rev. B75 (2007) 214405.

[33] F.-J. Jiang, F. Kämpfer, C. P. Hofmann, and U.-J. Wiese, Eur. Phys. J. 69 (2009) 473. 
[34] H. G. Evertz, G. Lana, and M. Marcu, Phys. Rev. Lett. 70 (1993) 875.

[35] A. W. Sandvik and H. G. Evertz, arXiv:0807.0682 [cond-mat.str-el].

[36] U. Gerber, C. P. Hofmann, F.-J. Jiang, M. Nyfeler, and U.-J. Wiese, J. Stat. Mech. (2009) $\mathrm{P} 03021$.

[37] F.-J. Jiang, F. Kämpfer, M. Nyfeler, and U.-J. Wiese, Phys. Rev. B78 (2008) 214406.

[38] M. Göckeler and H. Leutwyler, Nucl. Phys. B350 (1991) 228.

[39] M. Göckeler and H. Leutwyler, Phys. Lett. B253 (1991) 193. 